\title{
Primary malignant melanoma of the esophagus treated with subtotal esophagectomy: a case report
}

\author{
Shota Kuwabara, Yuma Ebihara*, Yoshitsugu Nakanishi, Toshimichi Asano, Takehiro Noji, Yo Kurashima, \\ Soichi Murakami, Toru Nakamura, Takahiro Tsuchikawa, Keisuke Okamura, Toshiaki Shichinohe and Satoshi Hirano
}

\begin{abstract}
Background: Primary malignant melanoma of the esophagus (PMME) is a rare disease with a poor prognosis. There are few reports of early-stage cases in which tumor invasion reached the lamina propria or muscularis mucosae, as in the present case. A standard treatment for early-stage PMME has not yet been established. The present study aimed to summarize previous reports and to discuss the indications for surgical treatment of early-stage primary malignant melanoma of the esophagus.
\end{abstract}

Case presentation: A 70-year-old woman with PMME was referred to our hospital. She underwent thoracoscopic and laparoscopic subtotal esophagectomy with lymphadenectomy. The resected specimen showed melanocytosis and junctional activity. Melanoma-specific antigens melan-A, S-100, and HMB45 were detected by immunohistochemical staining. The pathological diagnosis was pT1a-MM, pNO, pMO, and pStage IA. She remains alive without evidence of recurrence 39 months later.

Conclusion: Subtotal esophagectomy with regional radical lymphadenectomy could be recommended to patients with early-stage primary malignant melanoma of the esophagus, and curative surgical resection could improve their prognosis.

Keywords: Esophagus, Melanoma, Pathology, Treatment, Prognosis

\section{Background}

Primary malignant melanoma of the esophagus (PMME) is a rare disease. The incidence of PMME in all esophageal malignancies is low at $0.1 \%-0.2 \%$ [1]. The prognosis of PMME is poor because of its highly malignant biological behavior and its tendency to frequently disseminate even at the time of diagnosis. The recently reported 5 -year survival rate after surgical resection is $37.5 \%$ [2], which is lower than that of esophageal cancer. Given that PMME is a rare disease with a poor prognosis, an appropriate treatment of choice for PMME is still under investigation. Here, we present a case of early-stage PMME in which tumor invasion reached the muscularis mucosae that followed a favorable course after subtotal esophagectomy.

\footnotetext{
*Correspondence: yuma-ebi@wc4.so-net.ne.jp

Department of Gastroenterological Surgery II, Hokkaido University Graduate School of Medicine, North 15 West 7, Kita-ku, Sapporo, Hokkaido 0608638, Japan
}

(c) The Author(s). 2017 Open Access This article is distributed under the terms of the Creative Commons Attribution 4.0 International License (http://creativecommons.org/licenses/by/4.0/), which permits unrestricted use, distribution, and reproduction in any medium, provided you give appropriate credit to the original author(s) and the source, provide a link to the Creative Commons license, and indicate if changes were made. The Creative Commons Public Domain Dedication waiver (http://creativecommons.org/publicdomain/zero/1.0/) applies to the data made available in this article, unless otherwise stated.
A 70-year-old woman presented at her local hospital with a sticky sensation in her throat and a weight loss of $2 \mathrm{~kg}$ over 10 months.

Esophagogastroduodenoscopy (EGD) revealed an elevated lesion $35 \mathrm{~cm}$ from the incisors that was diagnosed as malignant melanoma by biopsy. She was referred to our institution for further examination and treatment. Her blood examination was normal, including tumor markers such as CEA and CA19-9, except for a slightly elevated HbA1c level.

A barium swallow test was arranged and showed a filling defect in the lower esophagus (Fig. 1). EGD showed a pigmented and elevated lesion $7 \mathrm{~mm}$ in diameter, associated with a hemi-circumferential, irregular-shaped, pigmented, and flat lesion in the lower esophagus. The flat lesion ranged from dark 


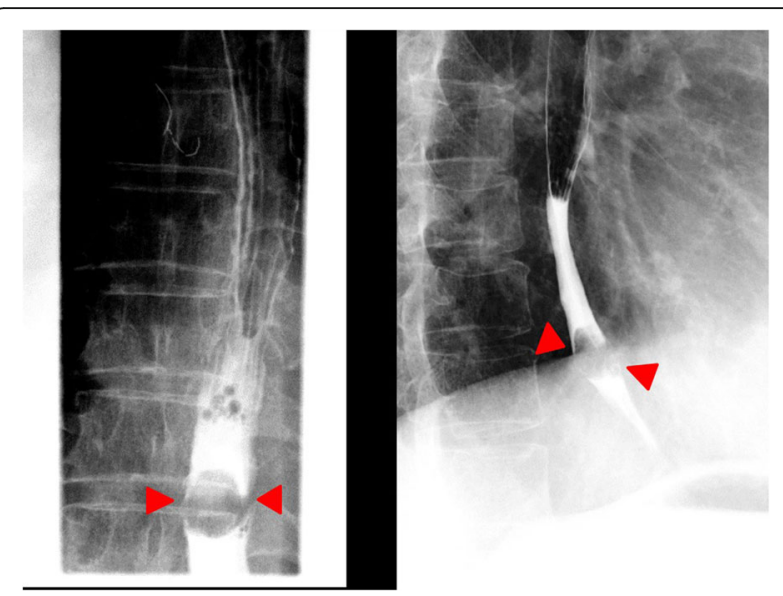

Fig. 1 Findings of a barium swallow test. A barium swallow test shows a filling defect in the lower esophagus (arrows)

brown to black in color, and the black area contained a well-demarcated mucosal abnormality (Fig. 2).

A biopsy specimen showed malignant melanoma cells in the esophageal mucosa, which were strongly positive for melanoma-specific antigens S-100 and HMB45 by immunohistochemical staining. A computed tomography $(\mathrm{CT})$ scan also showed an intraluminal mass in the lower esophagus, which was well-defined without infiltration into the surrounding tissues (Fig. 3a). There was no enlargement of mediastinal lymph nodes or any visible metastatic lesion. 18F-fluorodeoxyglucose (18FFDG) positron-emission tomography (PET) combined with CT showed abnormal 18F-FDG uptake in the

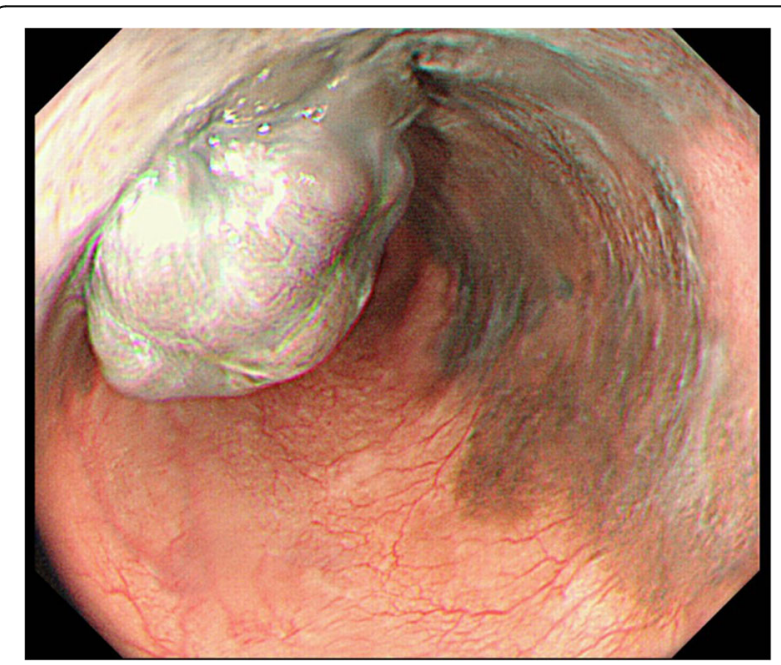

Fig. 2 Findings of esophagogastroduodenoscopy (EGD). EGD shows a pigmented, elevated 7-mm lesion, indicating an almost circumferential, irregular-shaped pigmentation in the lower esophagus, ranging from dark brown to black. The black area contains a well-demarcated mucosal abnormality same part of the esophagus identified on EGD and barium swallow as the site of the lesion with a maximum standardized uptake value (SUV max) of 3.1 (Fig. 3b). Detailed clinical examination of the eyes, oral cavity, nose, and skin did not indicate any malignant melanoma lesions.

Based on these findings, the preoperative diagnosis of the lesion was PMME without metastasis (cT2N0M0, cStage II) according to the UICC TNM classification of esophageal cancer [3]. Then, thoracoscopic and laparoscopic subtotal thoracic esophagectomy with lymphadenectomy of the neck, mediastinum, and abdomen was performed. The cervical esophagus and the elevated gastric tube were anastomosed via the posterior mediastinal approach. The operation time was $536 \mathrm{~min}$, and blood loss was $95 \mathrm{~mL}$.

The resected specimen showed an elevated, black, and pigmented polyp-like lesion $(15 \mathrm{~mm} \times 13 \mathrm{~mm} \times 9 \mathrm{~mm})$ in a flat and pigmented area $(57 \mathrm{~mm} \times 38 \mathrm{~mm})$ (Fig. 4a). A faintly marked depression that was partly tinged with white on the surface of the polyp-like lesion was found (Fig. 4b). Microscopic examination showed that most of the polyp-like lesion was composed of solid growth, with pseudocircular and spindle-shaped atypical cells containing melanin pigmentation and irregularly demarcated nucleoli. Many melanophages were present in the intervening interstitial stroma (Fig. 5). The lesion was immunohistochemically stained strongly for melanoma-specific antigens melan-A, S-100, and HMB45 (Fig. 6a-c). Around the polyp-like lesion, the same characteristic cells were spread laterally in the epithelium. Melanophages were also present in the lamina propria beneath the polyp-like lesion. The tumor cells were thought to invade the muscularis mucosae directly and then spread horizontally in the basal layer of the esophageal epithelium, which is called "junctional activity" [4] (Fig. 7a-c). The proximal and distal margins were considered safe. No lymph node metastases were detected. Pathologically, a diagnosis of pT1a-MM, pN0, pM0, pStage IA [3] was rendered.

Her postoperative course was uneventful and favorable. Adjuvant chemotherapy was not administered, and she has survived 39 months so far without any evidence of recurrence. She has been followed up once a half year and underwent blood tests and contrast-enhanced CT to search for metastasis or recurrence.

\section{Discussion and conclusions}

PMME is a rare disease with an extremely low incidence, comprising $0.1 \%-0.2 \%$ of all esophageal malignant tumors [1]. Over $70 \%$ of patients with PMME visit the hospital with chief complaints of dysphagia and epigastralgia [5]. Because the tumor is softer than other esophageal carcinomas, and wall extensibility of the 


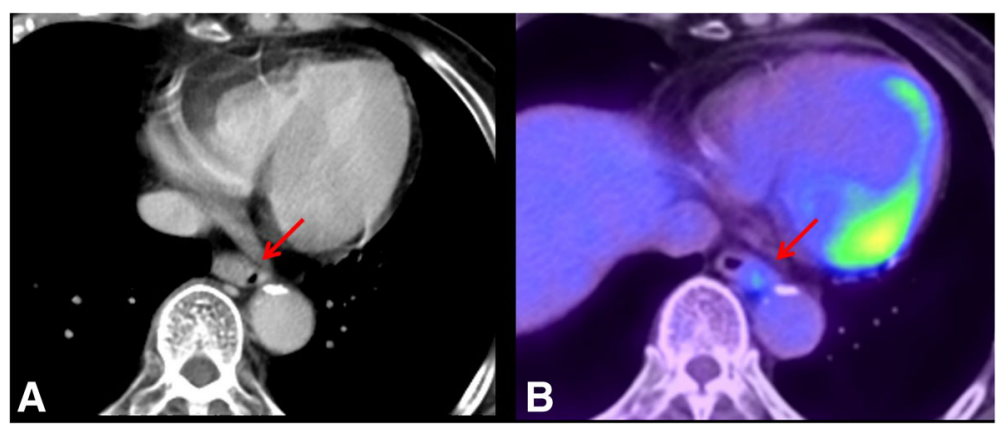

Fig. 3 Findings of computed tomography (CT) and 18F-fluorodeoxyglucose (18F-FDG) positron-emission tomography/computed tomography $(P E T-C T)$. a A transverse plane of an enhanced CT scan shows an intraluminal mass in the lower esophagus, which is well-defined without infiltration of the surrounding tissue (arrows). b 18F-FDG PET-CT scan shows 18F-FDG uptake at the same lesion of the lower esophagus; the SUV max of the lesion is 3.1 (arrows)

esophagus is maintained, the onset of symptoms is slow despite the size of the tumor. Therefore, more than $90 \%$ of the tumors are found to be larger than $2 \mathrm{~cm}$ at the initial diagnosis [5], and the detection of PMME at an early stage is rare.

Generally, PMME tends to originate in the lower to middle esophagus with endoscopic findings of a wellcircumscribed, elevated, and pigmented tumor that is partially covered by normal mucosa and rarely accompanied by ulcers. A black tone is well known as a characteristic of PMME, but various colors such as purple and brown are often present in 10\%-25\% of PMMEs depending on the melanin quantity $[1,6]$. The diagnosis of PMME should be suspected when a black or dark brown mass is observed [7]; however, it is important to be aware of amelanotic melanoma without white melanin pigmentation. Therefore, careful assessment is necessary for an accurate diagnosis [6] by endoscopy.

Esophageal melanocytosis is characterized by the presence of an increased number of pigment-laden melanocytes in the basal layer of the esophageal squamous epithelium, and the transfer of melanin granules to the epithelium around the melanocytes [8]. It has been described as a premalignant lesion of PMME; therefore, differentiation from melanoma in situ is important [9].
A biopsy can be conducted on patients for definitive diagnosis, but its accuracy is only approximately $80 \%$ [5]. Moreover, $20 \%-50 \%$ of patients are misdiagnosed with a poorly differentiated carcinoma [5], especially in cases of amelanotic melanoma. Immunohistochemical investigations are supportive for definitive diagnosis [10].

Diagnostic criteria are defined by Allen and Spitz [4] as follows: (1) a typical histological pattern of melanoma, with melanin granules inside the tumor cells, and an (2) origin in an area of junctional activity in the squamous epithelium. Junctional activity is defined as some nests of melanocytes with varying degrees of atypia found at the mucosal-submucosal junction adjacent to the tumor mass [4]. In other words, the tumor cells are spread horizontally in the basal layer of the esophageal epithelium. These findings and the presence of in situ melanoma without previous history of cutaneous melanoma lead to the absolute diagnosis of PMME [10]. In the present case, melanocytosis and junctional activity were surrounding the main tumor, and positive results of melan-A, S-100, and HMB45 were revealed by immunohistochemical staining, which led to a definitive diagnosis of PMME.

The prognosis of PMME seems to be improving because of the advances in endoscopic technology. In 1989,

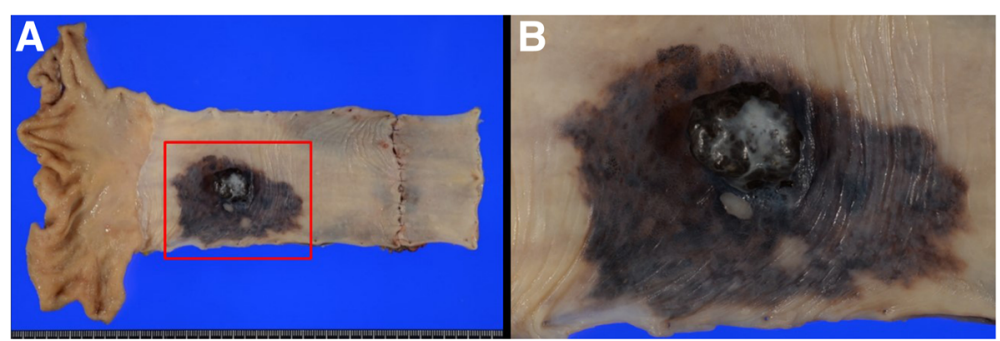

Fig. 4 Macroscopic findings. a The resected specimen showed an elevated, black-pigmented polyp-like lesion (15 mm $\times 13 \mathrm{~mm} \times 9 \mathrm{~mm})$ on a flat, black-pigmented area $(57 \mathrm{~mm} \times 38 \mathrm{~mm})$ in the lower esophagus. $\mathbf{b}$ A magnified image of the lesion (the part surrounded by a red square in Fig. 4a). There is a faintly marked depression partly tinged with white on the surface of the polyp-like lesion 


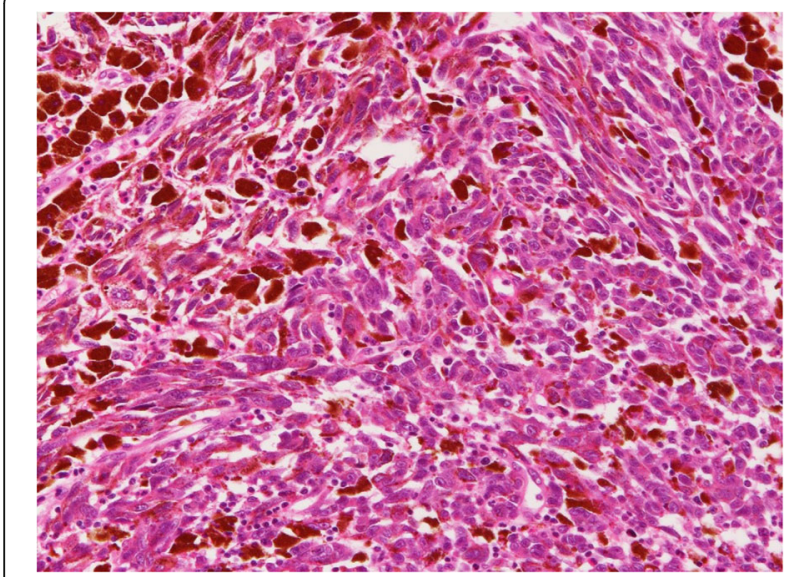

Fig. 5 Histopathological findings (hematoxylin and eosin (HE) staining). Most of the polyp-like lesion is composed of solid growth, pseudocircular, and spindle-shaped atypical cells containing melanin pigmentation and irregularly demarcated nucleoli. Many melanophages are present in the intervening interstitial stroma $(\mathrm{HE} \times 200)$

Sabanathan [5] reported that 5-year survival rate of PMME after surgery was $4.2 \%$, whereas Volpin et al. reported it was up to $37 \%$ in 2002 [11]. The increasing number of cases with early detection is one of the contributing factors of improving the prognosis [12]. However, the overall 5-year survival rate of advanced squamous esophageal carcinoma was reported to be $40 \%-50 \%$ [13], which is higher than PMME; therefore, the biological behavior of
PMME appears to be aggressive. Invasion deeper than T2 (hazard ratio: $2.288, p=0.0327,95 \%$ CI: 1.071-4.878) [14] and lymph node metastasis (hazard ratio: $15.05, p=0.013$, 95\% CI: 1.757-128.795) [15] have been reported as predictive factors for worse survival. On the contrary, Takahashi et al. [16] reviewed 33 patients with invasion depth of $\mathrm{T} 1 \mathrm{~b}$, pointed out the poor prognosis of T1b patients because of a high recurrence rate $(20$ of 33 patients), and reported that the 5-year survival rate was only $29.4 \%$.

Detection of the lesion in early stages seems to be relatively rare because of the characteristic delay in symptoms' appearance. We identified 10 previous T1a cases $[12,15,17-22]$ finally diagnosed by pathological tests, and their tumor location, size, depth of tumor, treatment, and outcomes were well-described. These cases were recorded from 1985 to 2015 and were found through literature search using the PubMed online database with "malignant melanoma" and "esophagus" as keywords (Table 1). In addition, no patients diagnosed in the early stage were reported in 1985-2000.

Among the 10 cases, subtotal esophagectomy with lymphadenectomy of the neck, mediastinal, and abdomen was performed in eight patients, and endoscopic mucosal resection (EMR) was performed in two patients because of the small tumor size. None of the cases received adjuvant therapy or had any signs of metastasis at diagnosis. Median disease-free survival time was 33 months and ranged from 15 to 94.7 months.

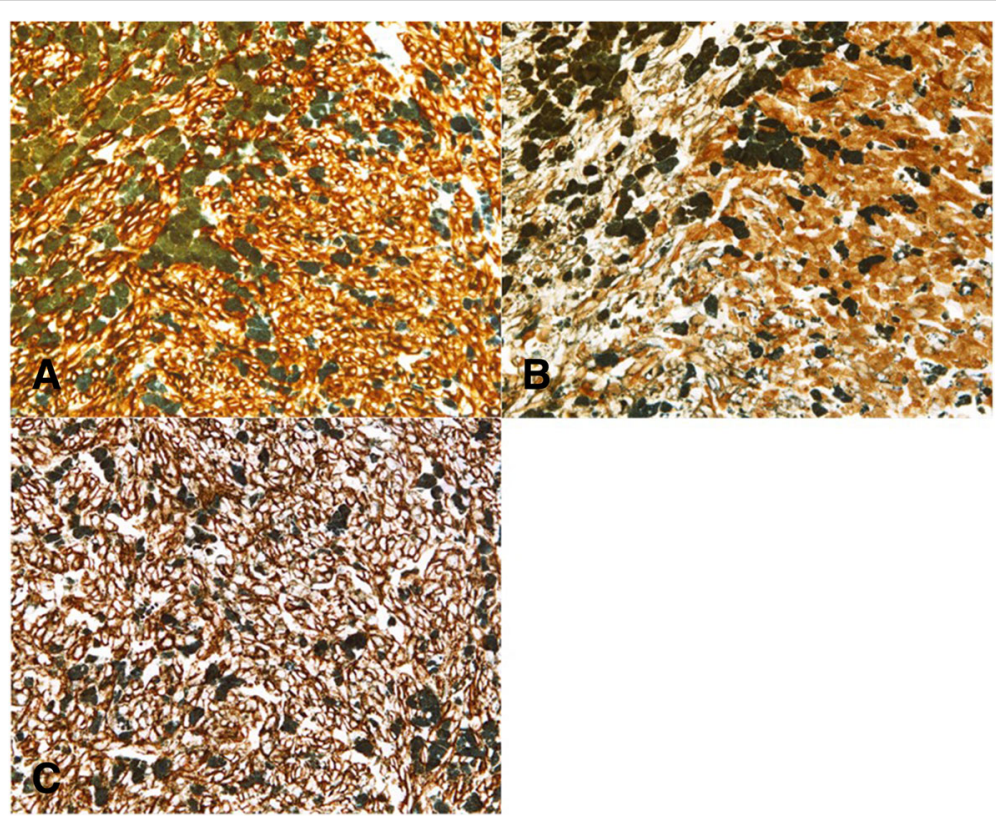

Fig. 6 Histopathological findings (immunohistochemical staining). Melanoma-specific antigens melan-A, S-100, and HMB45 are shown by immunohistochemical staining. a Melan-A $\times 200$, b S- $100 \times 200$, c HMB45 $\times 200$. The tumor cells are presented as brown pigment, and melanin is presented as green pigment 


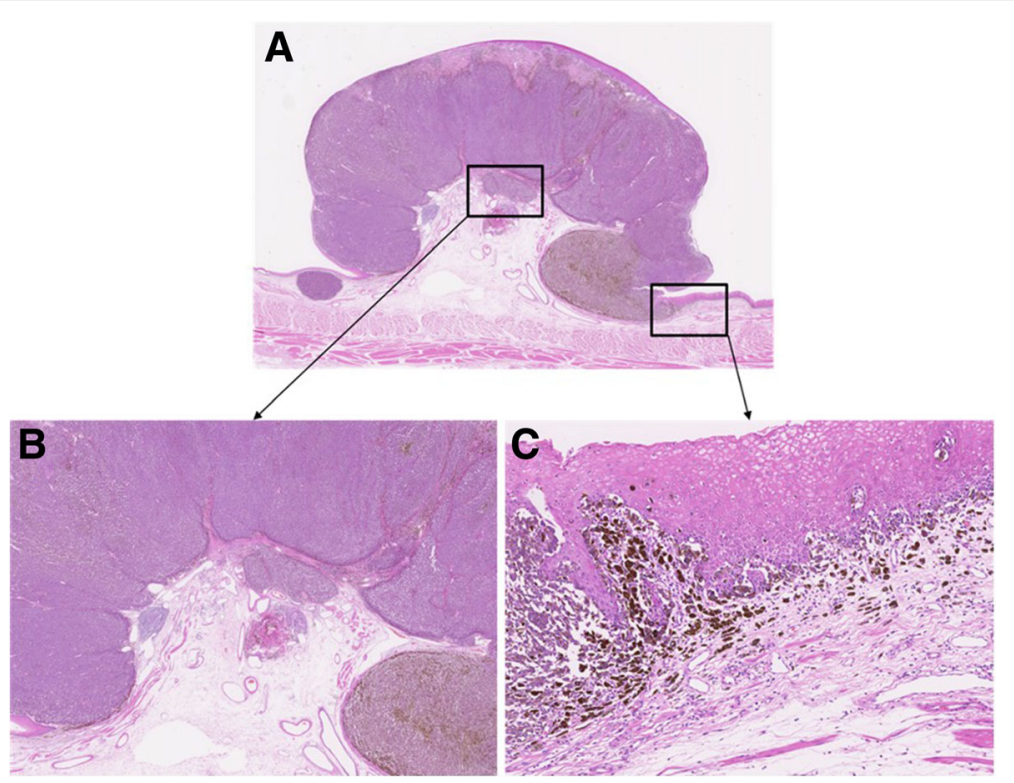

Fig. 7 Histopathological characteristics of the PMME (hematoxylin and eosin (HE) staining). a The PMME shows a polyp-like intraluminal mass (HE $\times 10)$. $\mathbf{b}$ The tumor cell invasion directly involves the muscularis mucosae $(H E \times 25)$. c The tumor cells are spread horizontally in the basal layer of the esophageal epithelium, which is considered junctional activity $(\mathrm{HE} \times 100)$

Treatment of PMME should be individualized for each patient. The choice should be based on tumor size and location, presence or absence of metastases, age, and comorbidities of the patients [23]. Kimura et al. [19] reported the first case of PMME treated by EMR and discussed the indications for EMR of superficial-type PMME. Miyatani et al. [12] reported that when the lesion was very small and a biopsy could not be obtained, EMR could be performed to obtain a definite diagnosis and to treat the patient. However, there have been few reports of PMME treated by EMR, and the indications for performing EMR should be evaluated cautiously after a detailed examination because of the risk of lymph node metastasis. Diagnosing PMME as T1a accurately is very difficult; therefore, we recommend subtotal esophagectomy with radical lymphadenectomy of the neck, mediastinum, and abdomen for treatment of PMME [D2]. Conversely, for patients with PMME at T1a, curative surgical resection could improve their prognosis. Although there is probably no absolute indication of adjuvant therapy for $\mathrm{T} 1 \mathrm{a}$ and negative lymphoid metastatic cases because of low risk of metastasis and recurrence, as with esophageal cancer, careful follow-up such as blood tests including tumor marker and image inspection using contrast-enhanced CT would be necessary. On the contrary, systemic chemotherapy based on

Table 1 Case reports of early primary malignant melanoma of the esophagus stage T1a (1985-2015)

\begin{tabular}{|c|c|c|c|c|c|c|c|c|}
\hline Author & Year & Age & Sex & Location & Size $(\mathrm{cm})$ & Depth $^{* a}$ & Treatment & Course $^{* b}$ \\
\hline Kido [16] & 2000 & 60 & $M$ & $\mathrm{Lt}$ & $4.0 \times 2.0$ & T1a & $C R$ & 33 months \\
\hline Mikami [17] & 2001 & 41 & $\mathrm{~F}$ & Mt & $0.8 \times 0.6$ & T1a & $C R$ & 31 months \\
\hline Kimura [18] & 2005 & 73 & M & Lt & $1.8 \times 1.3$ & T1a-LPM & EMR & 15 months \\
\hline Suzuki [19] & 2008 & 62 & M & Mt & $7.0 \times 4.5$ & T1a-EP & $C R$ & 33 months \\
\hline Suzuki [19] & 2008 & 67 & M & $\mathrm{Lt}$ & $5.5 \times 5.5$ & T1a-LPM & $C R$ & 53 months \\
\hline Miyatani [11] & 2009 & 64 & $\mathrm{~F}$ & $\mathrm{Lt}$ & 0.5 & T1a-LPM & EMR & 20 months \\
\hline Minami [20] & 2010 & 72 & M & $\mathrm{Lt}$ & unknown & T1a-EP & $C R$ & 25 months \\
\hline Wang [14] & 2013 & 62 & M & Mt & $7.0 \times 4.5$ & T1a & $C R$ & 93.7 months \\
\hline Yamamoto [21] & 2015 & 75 & M & $\mathrm{Lt}$ & $1.5 \times 1.0$ & T1a-MM & $C R$ & 83 months \\
\hline Our case & 2015 & 78 & $\mathrm{~F}$ & $\mathrm{Lt}$ & $5.7 \times 3.8$ & T1a-MM & $C R$ & 39 months \\
\hline
\end{tabular}

*a : According to the Japanese Classification of Esophageal Cancer, 11th Edition. Japan Esophageal Society Esophagus (2017). ${ }^{* b}$ : All reported cases are still alive after the treatment, and none have had any symptoms of relapse or distant metastasis

$M t$ middle of the esophagus, $L t$ lower esophagus, $E M R$ endoscopic mucosal resection, $C R$ curative resection (subtotal esophagectomy and radical lymphadenectomy of the neck, mediastinum, and abdomen) 
cutaneous malignant melanoma should be considered for deeper than $\mathrm{T} 1 \mathrm{~b}$ and positive lymphoid metastatic cases, but its efficacy in increasing overall survival of patients with PMME is still unknown. Meanwhile, neoadjuvant therapy for PMME has not been reported. Therefore, if the lesion was evaluated to be resectable at the time of diagnosis, curative surgical resection with radical lymphadenectomy of the neck, mediastinum, and abdomen [D2] could be performed.

We presented a rare case of early-stage PMME. A standard treatment for early-stage PMME has not yet been established, but subtotal esophagectomy with regional radical lymphadenectomy could be recommended for patients with PMME at T1a, and curative surgical resection could improve their prognosis. Further accumulation of cases is necessary to evaluate the relationship between treatment strategy and long-term prognosis.

\section{Abbreviations}

CA19-9: Carbohydrate antigen 19-9; CEA: Carcinoembryonic antigen; EGD: Esophagogastroduodenoscopy; EMR: Endoscopic mucosal resection; PMME: Primary malignant melanoma of the esophagus

\section{Acknowledgements}

We would like to thank Editage (www.editage.jp) for English language editing.

\section{Availability data and materials}

All data generated or analyzed during this study are included in this published article.

\section{Funding}

None

\section{Authors' contributions}

SK and YE wrote the manuscript; TS and SH revised the manuscript; YE, YK, $\mathrm{SM}$, and TS performed the surgery; $\mathrm{YN}, \mathrm{TA}, \mathrm{TNa}, \mathrm{TNO}, \mathrm{T}$, and $\mathrm{KO}$ acquired the data. All authors read and approved the final manuscript.

\section{Ethics approval and consent to participate}

Not applicable

\section{Consent for publication}

Written informed consent was obtained from the patient for publication of this case report and any accompanying images. A copy of the written consent is available for review by the Editor of this journal.

\section{Competing interests}

The authors declare that they have no competing interests relevant to this article.

\section{Publisher's Note}

Springer Nature remains neutral with regard to jurisdictional claims in published maps and institutional affiliations.

Received: 9 August 2017 Accepted: 21 November 2017

Published online: 02 December 2017

\section{References}

1. Joob AW, Haines GK 3rd, Kies MS, Shields TW. Primary malignant melanoma of the esophagus. Ann Thorac Surg. 1995;60:217-22.

2. Yu H, Huang $X, L i$ Y, et al. Primary malignant melanoma of the esophagus: a study of clinical features, pathology, management and prognosis. Dis Esophagus. 2011;24:109-13.

3. Sobin L, Gospodarowicz MK, Wittekind C. TMN classification of malignant Tumors, 7th ed. Hoboken: Wiley-Blackwell; 2010.
4. Allen AC, Spitz S. Malignant melanoma; a clinicopathological analysis of the criteria for diagnosis and prognosis. Cancer. 1953;6:1-45.

5. Sabanathan S, Eng J, Pradhan GN. Primary malignant of the esophagus. Am J Gastroenterol. 1989:84:1475-81.

6. Taniyama K, Suzuki H, Sakuramachi S, Toyoda T, Matsuda M, Tahara E. Amelanotic malignant melanoma of the esophagus: case report and review of the literature. Jpn J Clin Oncol. 1990;20:286-95.

7. Li Y-H, Xu L, Zou X-P. Primary malignant melanoma of the esophagus: a case report. World J Gastroenterol. 2014;20(10):2731-4.

8. Yamazaki K, Ohmori T, Kumagai Y, et al. Ultrastructure of oesophageal melanocytosis. Virchows Arch A Pathol Anat Histopathol. 1991;418:515-22.

9. Walter A, van Rees BP, Heijnen BH, van Lanschot JJ, Offerhaus GJ. Atypical melanocytic proliferation associated with squamous cell carcinoma in situ of the esophagus. Virchows Arch. 2000;437:203-7.

10. Iwanuma Y, Tomita N, Amano T, Isayama F, Tsurumaru M, Hayashi T, Kaijyama Y. Current status of primary malignant melanoma of the esophagus: clinical features, pathology, management and prognosis. J Gastroenterol. 2012:47:21-8.

11. Volpin E, Sauvanet A, Couvelard A, Belghiti J. Primary malignant melanoma of the esophagus: a case report and review of the literature. Dis Esophagus. 2002;15:244-9.

12. Miyatani H, Yoshida Y, Ushimaru S, Sagihara N, Yamada S. Slow growing flat-type primary malignant melanoma of the esophagus treated with capassisted EMR. Dig Endosc. 2009;21:255-7.

13. Ando N, Ozawa S, Kitajima Y. Improvement in the results of surgical treatment of advanced squamous esophageal carcinoma during 15 consecutive years. Ann Surg 2000;232:225-32.

14. Yamaguchi T, Shioaki Y, Koide K, et al. A case of primary malignant melanoma of the esophagus and analysis of 193 patients in Japan (in Japanese). Nihon Shokakibyo Gakkai Zassi. 2004;101:1087-94.

15. Wang S, Tachimori Y, Hokamura N, Igaki H, Kishino T, Kushima R. Diagnosis and surgical outcomes for primary malignant melanoma of the Esophagus: a single-Center experience. Ann Thorac Surg. 2013;96:1002-7.

16. Takahashi H, Ichikawa N, Takahashi S, Hirose K. Primary malignant of the esophagus of submucosal invasion: a case report. (in Japanese). Nihon Rinsyogeka Gakkai Zassi. 2013;74(6):1473-8.

17. Kido T, Morishima H, Nakahara M, Nakao K, Tanimura H, Nishimura R, Tsujimoto M. Early stage primary malignant melanoma of the esophagus. Gastrointest Endosc. 2000;51(1):90-1.

18. Mikami T, Fukuda S, Shimoyama T, Yamagata R, Nishiya D, Sasaki Y, Uno Y, Saito H, Takaya S, Kamata Y, Munataka A. A case of early-stage primary malignant melanoma of the esophagus. Gastrointest Endosc. 2001;53(3):365-7.

19. Kimura H, Kato H, Sohda M, Nakajima M, Fukai Y, Miyazaki T, Masuda N, Manda R, Fukuchi M, Ojima H, Tsukada K, Kuwano H. Flat-type primary malignant melanoma of the esophagus treated by EMR: case report. Gastrointest Endosc. 2005;61 (6):787-9.

20. Suzuki $H$, Nakanishi $Y$, Taniguchi $H$, Shimoda T, Yamaguchi $H$, Igaki $H$, Tachimori Y, Kato H. Two cases of early-stage esophageal malignant melanoma with long-term survival. Pathol Int. 2008:58:432-5.

21. Minami H, Inoue $H$, Satodate $H$, Hamatani $\mathrm{S}$, Kudo S. A case of primary malignant melanoma in situ in the esophagus. Gastrointest Endosc. 2011; 73(4):814-5.

22. Yamamoto S, Makuuchi H, Kumaki N, Ozawa S, Shimada H, Chino O, Kazuno A, Yasuda S, Tamayama T, Sakai I. A long surviving case of multiple early stage primary malignant melanoma of the esophagus and a review of the literature. Tokai J Exp Clin Med. 2015;40(3):90-5.

23. Machado J, Ministro P, Araujo R, Cancela E, Castanheira A, Silva A. Primary malignant melanoma of the esophagus: a case report. World I Gastroenterol. 2011;17(42):4734-8. 